\title{
Hyperbolic Hydrodynamical Model of Carrier Transport in Semiconductors
}

\author{
ANGELO MARCELLO ANILE ${ }^{a}$, VITTORIO ROMANO ${ }^{b, *}$ and GIOVANNI RUSSO ${ }^{c}$ \\ a Dipartimento di Matematica, Universitá di Catania, viale A. Doria, 6-95125 Catania, Italy; \\ ${ }^{\mathrm{b}}$ Politecnico di Bari, sede di Taranto, Viale del Turismo 8-74100 Taranto, Italy; \\ ${ }^{\mathrm{c}}$ Università dell'Aquila, Via Vetoio, loc. Coppito - 67100 L'Aquila
}

\begin{abstract}
A hydrodynamical model for semiconductors based on Extended Thermodynamics is presented and a suitable numerical scheme is proposed.
\end{abstract}

Keywords: TCAD, VLSI, BTE, transport theory, fluid mechanics, electronic devices

\section{INTRODUCTION}

Hydrodynamical models for carrier transport in semiconductors have been widely investigated by several authors $[1,2,3,4,5,6$ and al.]. Most hydrodynamical models are based on phenomenological assumptions and not derived from a consistent physical model. Here we present a hydrodynamical model based on Extended Thermodynamics which is the appropriate thermodynamic theory of irreversible processes far from thermodynamic equilibrium. The model is obtained from the set of moment equations of the Boltzmann transport equation for electrons at the level of 13 moments. The closure relations are obtained in the framework of Extended Thermodynamics $[7,8]$ by employing the entropy principle. The balance equations for the macroscopic variables form a hyperbolic system for values of heat flux and stress tensor which are routinely encountered in the simulation of semiconductor devices of interest for industrial purposes. In the present paper we model the productions as relaxation terms, with relaxation times functions of the moments.

A suitable numerical scheme, developed in [10], has been successfully applied to the present model. It is based on the splitting method between relaxation and convection and allows an easy implementation in numerical codes. For the convective steps we used the Nessyahu-Tadmor [11] scheme for quasilinear hyperbolic systems in divergence form. It is a second order shock capturing method and does not require the analytical expressions of the eigenvalues and eigenvectors of the Jacobian matrix of fluxes.

${ }^{*}$ Corresponding author. 
The latter property is crucial because explicit expressions for the eigenvalues and eigenvectors are not available for the balance equations of Extended Thermodynamics. For the relaxation part a suitable combinations of implicit Euler steps assures a global second order accuracy.

In particular we present the simulation of a $n^{+}-n-n^{+}$silicon diode.

The numerical results shows a uniform accuracy in all the fields (energy density, velocity, particle number, heat flux) within a reasonable degree of tolerance and could be employed for a systematic use as a CAD tool.

\section{MATHEMATICAL MODEL}

In the one dimensional case the evolution equations become (see [9] for the complete derivation)

$$
\begin{gathered}
\frac{\partial n}{\partial t}+\frac{\partial}{\partial x}(n v)=0, \\
\frac{\partial}{\partial t}(n v)+\frac{\partial}{\partial x}\left(n v^{2}+\frac{p}{m^{*}}+\frac{\sigma}{m^{*}}\right)=-\frac{n v}{\tau_{p}}-\frac{n e E}{m^{*}}, \\
\frac{\partial}{\partial t}\left(n v^{2}+\frac{3 p}{m^{*}}\right)+\frac{\partial}{\partial x}\left(n v^{3}+\frac{5 v p}{m^{*}}+\frac{2 \sigma v}{m^{*}}+\frac{2 q}{m^{*}}\right) \\
=-2 \frac{W-W_{0}}{m^{*} \tau_{\omega}}-\frac{2 n e v E}{m^{*}}, \\
\frac{\partial}{\partial t}\left(\frac{2}{3} n v^{2}+\frac{\sigma}{m^{*}}\right)+\frac{\partial}{\partial x}\left(\frac{2}{3} n v^{3}+\frac{4}{3} \frac{v p}{m^{*}}+\frac{7}{3} \frac{v \sigma}{m^{*}}+\frac{8}{15} \frac{q}{m^{*}}\right) \\
=-\frac{1}{m^{*} \tau_{s}}\left(n v^{2}+\frac{\sigma}{m^{*}}\right)-\frac{4 n e v E}{3 m^{*}} \\
\frac{\partial}{\partial t}\left(n v^{3}+\frac{5 v p}{m^{*}}+\frac{2 \sigma v}{m^{*}}+\frac{2 q}{m^{*}}\right) \\
+\frac{\partial}{\partial x}\left[n v^{4}+5 \frac{p^{2}}{n\left(m^{*}\right)^{2}}+7 \frac{\sigma p}{n\left(m^{*}\right)^{2}}+\frac{32}{5} \frac{q v}{m^{*}}\right. \\
\left.+v^{2}\left(8 \frac{p}{m^{*}}+5 \frac{\sigma}{m^{*}}\right)+\frac{148}{25} \frac{q^{2}}{m^{*} p}\right]
\end{gathered}
$$

$$
\begin{aligned}
= & -\frac{1}{\tau_{q}}\left(n v^{3}+\frac{5 v p}{m^{*}}+\frac{2 \sigma v}{m^{*}}+\frac{2 q}{m^{*}}\right) \\
& -\frac{e E}{m^{*}}\left(3 n v^{2}+\frac{5 p}{m^{*}}+\frac{2 \sigma}{m^{*}}\right) .
\end{aligned}
$$

In the previous equations, $n$ is the electron number density, $v$ the mean velocity, $p=n k_{\mathrm{B}} T$ the hydrostatic pressure, $q$ the $x$-component of the heat flux, $\sigma$ the $x x$-component of the viscosity tensor, $m^{*}$ the effective electron mass in the parabolic band approximation and $E$ the $x$ component of the electric field

$$
\begin{gathered}
E=\frac{\partial \phi}{\partial x} \\
\varepsilon \frac{\partial^{2} \phi}{\partial x^{2}}=-e\left(N_{\mathrm{D}}-N_{\mathrm{A}}-n\right),
\end{gathered}
$$

where $\phi$ is the electric potential, $N_{\mathrm{D}}$ and $N_{\mathrm{A}}$ are respectively the donor and acceptor density, $e$ the elemetary charge and $\varepsilon$ the dielectric constant.

$W$ is the electron energy density $W=(1 / 2)$ $n v^{2}+(3 / 2) n k_{\mathrm{B}} T$ and $W_{0}=(3 / 2) n k_{\mathrm{B}} T$. For the energy relaxation time, $\tau_{w}$, and viscous relaxation timei, $\tau_{\sigma}$, we take the constant values $\tau_{w}=0.47 \mathrm{ps}$ and $\tau_{\sigma}=0.02 \mathrm{ps}$. The relaxation times of momentum and energy flux have been obtained by the homogeneous Monte Carlo [12] data by using the following fitting formulas (see [9] for the value of the constants $c_{i}$ and $\tilde{c}_{i}$ )

$$
\begin{aligned}
\tau_{p} & =c_{1}+c_{2} \frac{S}{J}+c_{3} \frac{S^{2}}{J^{2}}, \\
\tau_{q} & =\tilde{c}_{1}+\tilde{c}_{2} \frac{S}{J}+\tilde{c}_{3} \frac{S^{2}}{J^{2}} .
\end{aligned}
$$

System $(1-5)$ has the structure of a hyperbolic system of balance laws. Hyperbolicity, however, is restricted to a suitable neighborhood of thermodynamical equilibrium (see [9]).

Numerical solutions of the system are obtained by a splitting scheme. Let us consider a system of the form

$$
\frac{\partial v}{\partial t}+\frac{\partial F(v)}{\partial x}=G(v)
$$

with $v \in \mathbf{R}^{m}$ and $F: \mathbf{R}^{m} \rightarrow \boldsymbol{R}^{m}$. 
The splitting scheme is based on the solution of the two steps:

Convection step

$$
\frac{\partial v}{\partial t}+\frac{\partial F}{\partial v}=0
$$

Relaxation step

$$
\frac{\partial v}{\partial t}=G(v)
$$

Each convection step is a predictor-corrector scheme of the form

$$
\begin{gathered}
v_{j+1 / 2}^{n+1 / 2}=\frac{1}{2}\left(v_{j}^{n+1}+v_{j+1}{ }^{n}\right)+\frac{1}{8}\left(v_{j}^{\prime}-v_{j+1}^{\prime}\right) \\
-\lambda\left[F\left(v_{j+1}^{n+1 / 2}\right)-F\left(v_{j}^{n+1 / 2}\right)\right] \\
v_{j}^{n+1 / 2}=v_{j}^{n}-\frac{\lambda}{2} F^{\prime}{ }_{j} .
\end{gathered}
$$

where $\lambda=d t / d x$. The time step $\Delta t$ must satisfy a stability condition

$$
\lambda \cdot \max \rho(A(v(x, t)))<\frac{1}{2} .
$$

where $\rho(A(v(x, t)))$ is the spectral radius of the Jacobian matrix,

$$
A=\frac{\partial F}{\partial v}
$$

This condition will ensure that the generalized Riemann problems with piecewise smooth data at time $t_{n}$ will not interfere during the time step $\Delta t$.

The values of $v^{\prime}{ }_{j} / \Delta x$ and $F_{j}^{\prime} / \Delta x$ are a first order approximation of the space derivatives of the field and of the flux, computed from cell averages by using Uniform Non Oscillatory reconstruction (UNO),

$$
\begin{aligned}
v_{j}^{\prime}= & \operatorname{MM}\left(d_{j-\frac{1}{2}} v+\frac{1}{2} \operatorname{MM}\left(D_{j-1}, D_{j}\right), d_{j+\frac{1}{2}} v\right. \\
& \left.-\frac{1}{2} \operatorname{MM}\left(D_{j}, D_{j+1}\right)\right)
\end{aligned}
$$

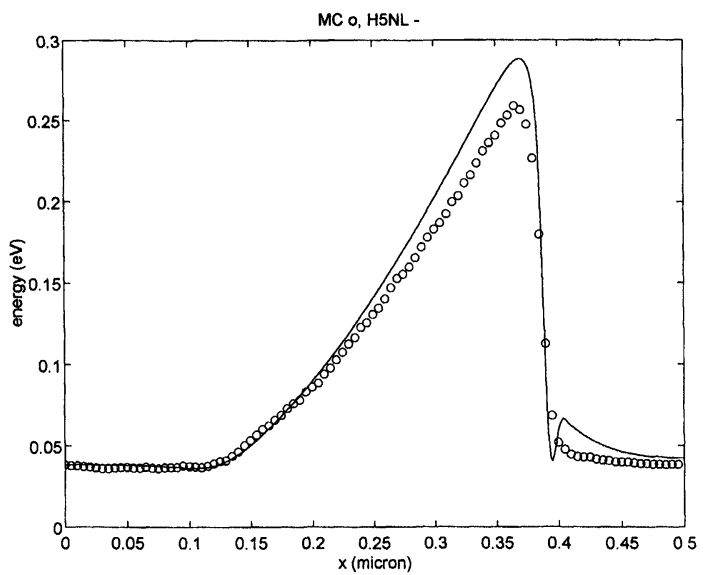

FIGURE 1 Average electron energy vs. distance obtained with the hydrodynamical model (continuous line) compared to Monte Carlo results (circles).

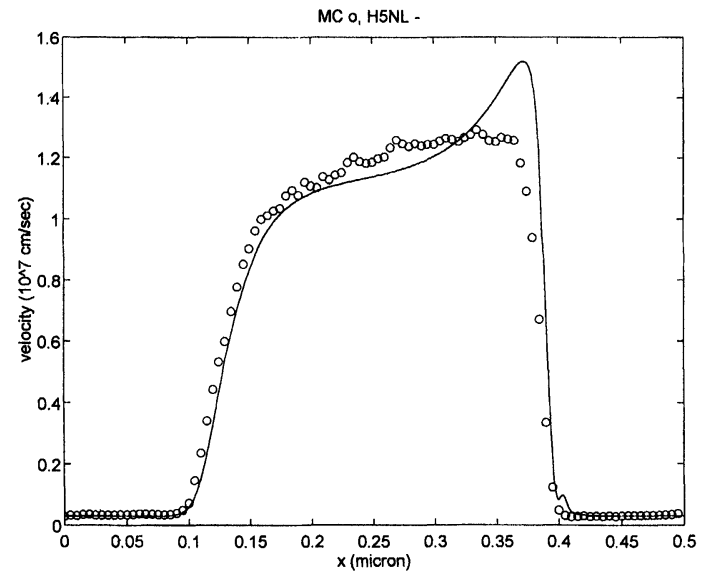

FIGURE 2 As in Figure 1 for the average drift velocity vs. distance.

where

$$
\begin{gathered}
D_{j}=v_{j+1}-2 v_{j}+v_{j-1}, \\
d_{j+1 / 2}=v_{j+1}-v_{j}
\end{gathered}
$$

and

$$
\begin{aligned}
& \operatorname{MM}(x, y)= \\
& \begin{cases}\operatorname{sgn}(x) \cdot \min (|x|,|y|) & \text { if } \operatorname{sgn}(x)=\operatorname{sgn}(y) \\
0 & \text { otherwise. }\end{cases}
\end{aligned}
$$

A similar procedure is used for computing $F^{\prime}{ }_{j}$. 
For the relaxation step an unconditionally stable second order scheme can be obtained by analytical integration of the linearized relaxation equation, where linearization is obtained by freezing the coefficients at time $t_{n}$. The electric potential is computed by a standard procedure. In order to obtain second order accuracy in time we combinine the two steps according to the following scheme

$$
\begin{aligned}
U_{1} & =U^{n}-R\left(U_{1}, E^{n}, \Delta t\right), \\
U_{2} & =\frac{3}{2} U^{n}-\frac{1}{2} U_{1}, \\
U_{3} & =U_{2}-R\left(U_{3}, E^{n}, \Delta t\right), \\
U_{5} & =\mathcal{C}_{\Delta t} U_{3}, \\
E^{n+1} & =\mathcal{P}\left(U_{4}\right), \\
U^{n+1} & =U_{4}-R\left(U^{n+1}, E^{n+1}, \Delta t / 2\right)
\end{aligned}
$$

where $R$ represents the discrete operator correspoding to the relaxation step, $\mathcal{C}_{\Delta t}$ is the discrete operator corresponding to NT scheme, and $\mathcal{P}(U)$ gives the solution to Poisson's equation.

\section{NUMERICAL RESULTS}

As test problem we consider a ballistic diode $n^{+}-n$ $n^{+}$, which models a MOSFET channel. The diode is made of silicon, and the bulk temperature is supposed to be $300 \mathrm{~K}$. The $n^{+}$regions are $0.1 \mu \mathrm{m}$ long and the channel is $0.3 \mu \mathrm{m}$ long. The doping density is $N_{D}^{+}=0.5 \times 10^{17} \mathrm{~cm}^{-3}$ in the $n^{+}$regions and $N_{D}=0.2 \times 10^{16} \mathrm{~cm}^{-3}$ in the channel.

For the electron effective mass in the approximation of parabolic band we use $m^{*}=0.32 m_{\mathrm{e}}$ when $m_{\mathrm{e}}$ is the electron mass. The silicon dielectric constant is given by $\varepsilon=\varepsilon_{r} \varepsilon_{0}$, where $\varepsilon_{r}=11.7$ is the relative dielecric constant and $\varepsilon_{0}=8.85 \times 10^{-18} \mathrm{C} /$ $\mathrm{V} \mu \mathrm{m}$ is the dielectric constant of vacuum.

The initial electron temperature is the lattice temperature $T_{0}=300 \mathrm{~K}$ and the charges are at rest. A bias voltage of 1 Volt is applied, and this determines a charge flux in the semiconductor.
The initial conditions for the system are;

$$
\begin{gathered}
n(x, 0)=n_{0}(x), \quad T(x, 0)=300 \mathrm{~K}, \quad v(x, 0) \\
=0, \quad q(x, 0)=0, \quad \sigma(x, 0)=0 .
\end{gathered}
$$

The doping profile is regularized according to the function

$$
n_{0}(x)=n_{0}-d_{0}\left(\tanh \frac{x-x_{1}}{s}-\tanh \frac{x-x_{2}}{s}\right),
$$

where $n_{0}=n_{0}(0), d_{0}=n_{0}\left(1-N \mathrm{D} / N_{\mathrm{D}}^{+}\right) / 2$, $x_{1}=0.1 \mu \mathrm{m}$, and $x_{2}=0.4 \mu \mathrm{m}$.

For the boundary conditions see [9]. The stationary solution is reached within a few picosends. The numerical results obtained for the stationary case have been compared with the Monte Carlo results, obtained by the DAMOCLES code [13].

Comparison with MC simulations shows a good overall agreement. In particular the agreement is very good everywhere in the device but in the second junction, where the discrepancy with MC simulations can reach up to $10 \%$.

\section{References}

[1] Haensh, W. (1991). The drift-diffusion equation and its application in MOSFET modeling, Springer-Verlag, Wien.

[2] Markowich, A., Ringhofer, C. A. and Schmeiser, C. (1990). Semiconductor Equations, Springer-Verlag, Wien.

[3] Blotekjaer, K. (1970). IEEE Trans. on Electron Devices, ED-17, 38.

[4] Baccarani, G. and Wordeman, M. R. (1982). Solid-state Electronics, 29, 970.

[5] Stettler, M. A., Alam, M. A. and Lundstrom, M. S. (1993). IEEE Trans. on Electron devices, 40(4), 733.

[6] Lee, S.-C. and Tang, T.-W. (1992). Solid-State Electronics, 35(4), 561 .

[7] Müller, I. and Ruggeri, T. (1993). Extended Thermodynamics, Springer-Verlag, Berlin.

[8] Jou, D., Casas-Vazquez e, J. and Lebon, G. (1993) Extended Irreversible Thermodynamics, Springer-Verlag, Berlin.

[9] Anile, A., Romano, V. and Russo, G. Extended Hydrodynamical Models of Carrier Transport in Semiconductors, preprint.

[10] Romano, V. and Russo, G. (1996). Numerical solution for hydrodynamical models of semiconductors, submitted to IEEE trans. on CAD.

[11] Nessyahu e, H. and Tadmor, E. (1990). J. Comput. Phys., 87(2), 408. 
[12] Anile, A. M. and Muscato, O. Improved hydrodynamical model for carrier transport in semiconductors, Phys. Rev. B., 51, 16728 .

[13] Fischetti, M. V. and Laux, S. (1993). Phys. Rev. B., 48, 2244.

\section{Authors' Biographies}

Angelo Marcello Anile is full professor of Mathematical Physics at Catania University (Italy). His research interests include mathematical models of semiconductors, nonlinear wave propagation, fluid dynamics, plasma physics, general relativity.

Vittorio Romano is assistent professor at Polytechnic of Bari (Italy). His research interests include mathematical models for semiconductors, asymptotic wave, relativistic fluids, radiation hydrodynamics.

Giovanni Russo is associate professor at the University of L'Aquila (Italy). His research interests include numerical analysis, mathematical models for semiconductors, wave propagation. 

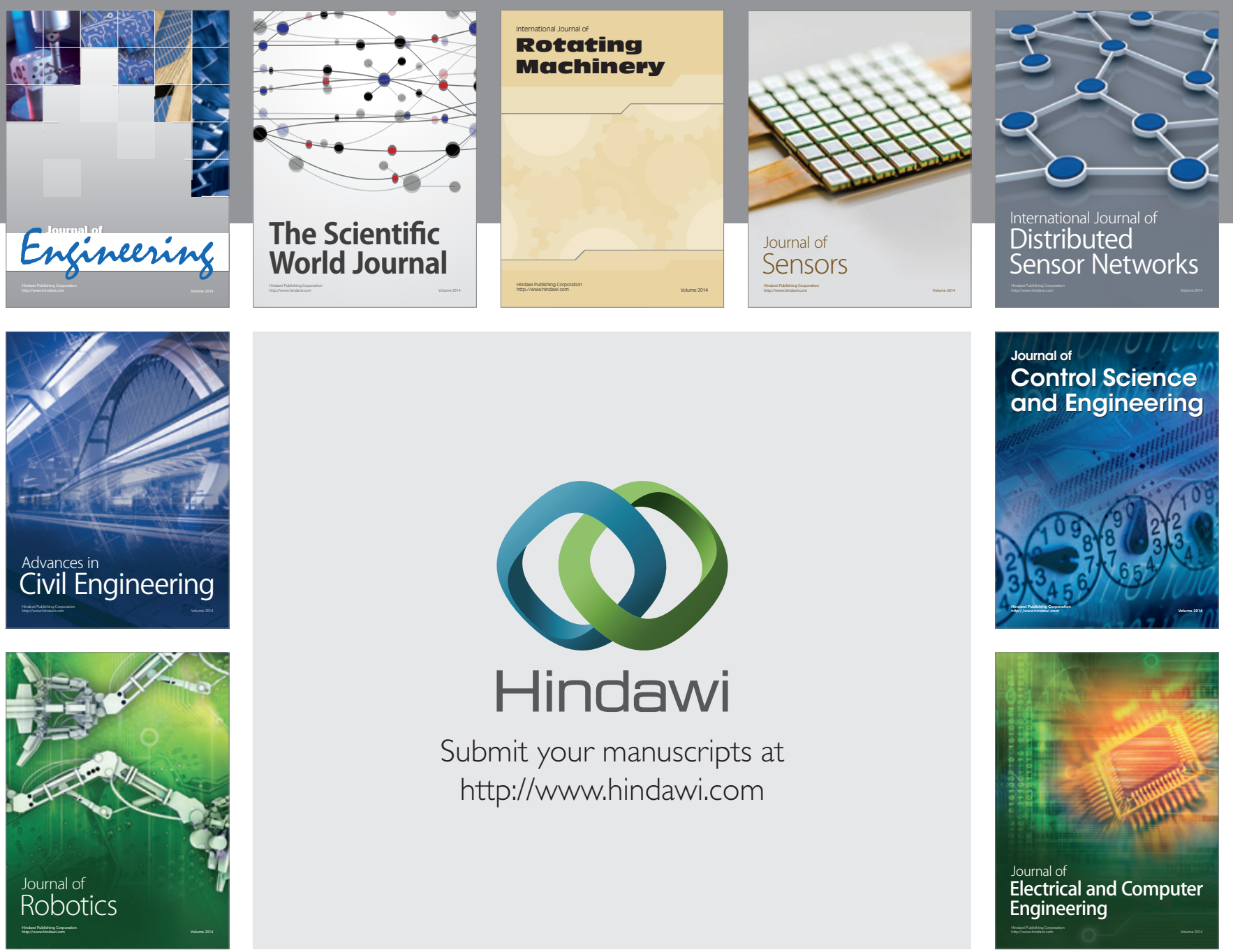

Submit your manuscripts at

http://www.hindawi.com
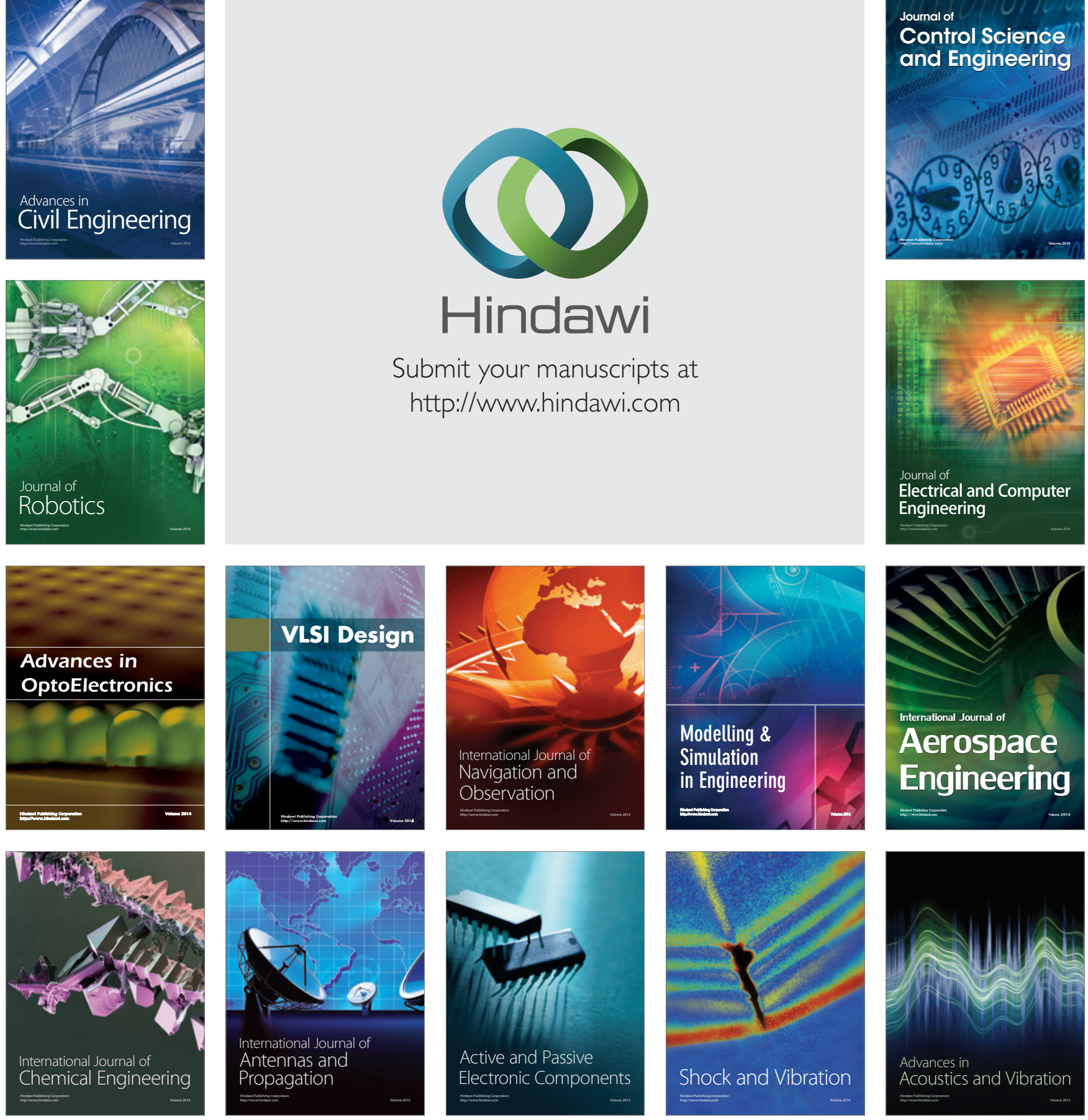